\title{
Detection of genetically modified organisms by electrochemiluminescence PCR method
}

\author{
Jinfeng Liu, Da Xing*, Xingyan Shen, Debin Zhu \\ Institute of Laser Life Science, South China Normal University, Guangzhou 510631, China
}

Received 9 December 2003; received in revised form 26 March 2004; accepted 26 March 2004

Available online 18 May 2004

\begin{abstract}
With the development of biotechnology, more and more genetically modified organisms (GMOs) have entered commercial market. Because of the safety concerns, detection and characterization of GMOs have attracted much attention recently. In this study, electrochemiluminescence polymerase chain reaction (ECL-PCR) combined with hybridization technique was applied to detect the GMOs in genetically modified (GM) soybeans and papayas for the first time. Whether the soybeans and the papayas contain GM components was discriminated by detecting the Cauliflower mosaic virus 35S (CaMV35S) promoter. The experiment results show that the detection limit for CaMV35S promoter is 100 fmol, and the GM components can be clearly identified in GM soybeans and papayas. The technique may provide a new means in GMOs detection due to its simplicity and high efficiency.
\end{abstract}

(C) 2004 Elsevier B.V. All rights reserved.

Keywords: ECL; GMO; Soybean; Papaya; CaMV35S promoter

\section{Introduction}

A genetically modified organism (GMO) is a living organism with its genome modified by the introduction of an exogenous gene. The gene is able to express an additional protein that confers new characteristics, i.e. herbicide tolerance or resistance to virus, antibiotic and insect (Niederhauser et al., 1996; Droge et al., 1998; Vollenhofer et al., 1999; Hails, 2000; Minunni et al., 2001; Mariotti et al., 2002). The foreign DNA is usually inserted into a gene 'cassette' consisting of an expression promoter $(\mathrm{P})$, a structural gene (encoding region) and an expression terminator (T). Two particular sequences are inserted into most of the available transgenic products: the promoter of the subunit $35 \mathrm{~S}$ of ribosomal RNA of cauliflower mosaic virus (CaMV35S) and the Nos terminator (Tnos) from Agrobacterium tumefaciens. In practice, they are widely used in the commercial production of various transgenic vegetables under brand names such as Roundup Ready for soy, Maigard for maize and the Flaver Savr for tomato (Mannelli et al., 2003).

\footnotetext{
* Corresponding author. Tel.: +86-20-85210089; fax: +86-20-85216052.

E-mail address: xingda@scnu.edu.cn (D. Xing).
}

Many countries have developed laws controlling the marketing of GMOs. At present, in the European Union (EU), labeling is mandatory for food product that contains ingredients derived from genetically modified maize (the Bt-Maize from Novartis) and soybean (RR-Soy from Monsanto) in percentage higher than $1 \%$ (Council Regulation (EC), 2000).

Methods for the identification of GMOs can be divided into three categories. The first category includes nucleotide-base amplification methods, such as polymerize chain reaction (PCR), ligase chain reaction (LPR), nucleic acid sequence-based amplification (NASBA), fingerprinting techniques (such as restriction fragment length polymorphism (RFLP), amplified fragment length polymorphism (AFLP), and random amplified polymorphic DNA (RAPD)), and probe hybridization. The second category includes protein-based methods, such as one-dimensional SDS gel electrophoresis, two-dimensional SDS gel electrophoresis, Western blot analysis and enzyme-linked immunosorbant assay (ELISA). The third category is based on the detection of enzymatic activities. Naturally, every detection method has its own specificities and limitations. The detection using an enzymatic activity method is not recommended for processed foods in which proteins may be denaturized. The methods based on PCR are not suitable for detection of 


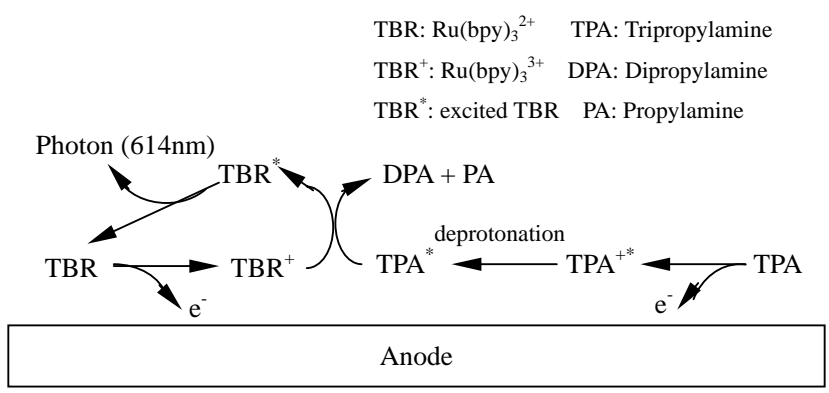

Fig. 1. Mechanism of ECL excitation. TBR and TPA are oxidized at the anode surface and form $\mathrm{TBR}^{+}$and $\mathrm{TPA}^{+*}$, respectively. The $\mathrm{TPA}^{+*}$ spontaneously loses a proton to form TPA*. The TPA*, a strong reductant, reacts with $\mathrm{TBR}^{+}$, a strong oxidant, to form the excited state of the label, TBR*. The excited state decays to the ground state through a normal fluorescence mechanism, emitting a photon at $614 \mathrm{~nm}$.

highly processed foods because DNA fragments in foods could be broken into pieces. Among the three categories, PCR is the most popular method used worldwide (Lin et al., 2001).

Electrochemiluminescence (ECL), where light-emitting species are produced by reactions between electrogenerated intermediates, has become an important and powerful analytical tool in recent years. An ECL reaction using tri-propylamine (TPA) and tris-(2,2'-bipyridyl) ruthenium (TBR) has been demonstrated to be a highly sensitive detection method for quantifying amplified DNA (Leland and Powell, 1990; Blackburn et al., 1991). A previously proposed ECL reaction for TBR + TPA is schematically shown in Fig. 1 (Hsueh et al., 1996; Blackburn et al., 1991; Deaver, 1995).

We employed ECL in GMOs detection because of its high sensitivity. For the first time, ECL, PCR and hybridization were combined to develop a sensitive method to detecting GMOs. In detail, the PCR products of sample (GMOs or non-GMOs) were mixed with a pair of probes designed specially to hybridize with $35 \mathrm{~S}$ promoter sequence, which is the characteristic of GMOs. After hybridization, the PCR products caught by the probes were collected and then the luminescence signal was detected using the ECL system, and according to the signal we can tell whether the sample was GMOs or not. In this study, we found the ECL signals of GMOs were much higher than those of non-GMOs.

\section{Materials and methods}

\subsection{Materials}

\subsubsection{Apparatus}

A custom-built ECL detection system is described in detail in our previous research (Zhu et al., 2003) (Fig. 2). The instrument is composed of an electrochemical reaction cell, a potentiostat (Sanming Fujian HDV-7C), an ultra high sensitivity single photon counting module (Channel Photomul-

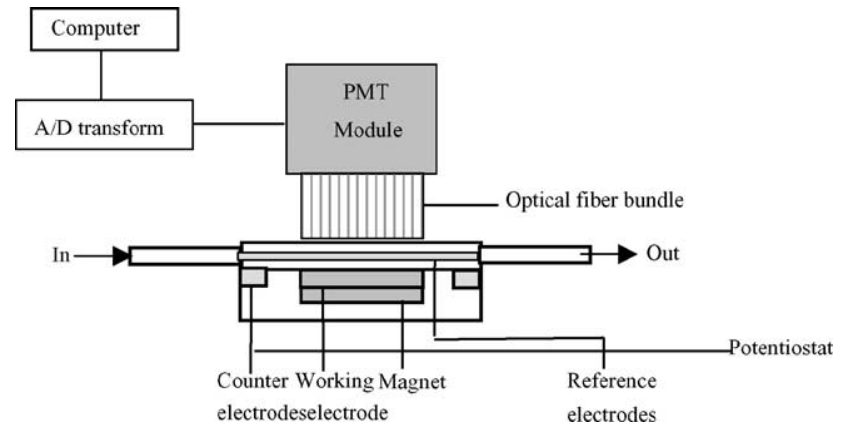

Fig. 2. Schematic of the ECL detection system. The signal from the PMT was amplified and discriminated. The transistor-transistor logic (TTL) pulses were counted with a multi-function acquisition card controlled by Labview software. The voltage applied to the electrodes was controlled with a potentiostat. The signal collection process and data analysis were accomplished with a personal computer.

tiplier, Perkin-Elmer MP-962), a multi-function acquisition card (Advantech PCL-836), a computer and labview software. The electrochemical reaction cell contains a working electrode (platinum), a counter electrode (platinum) and a reference electrode $\left(\mathrm{Ag} / \mathrm{AgCl}_{2}\right)$.

\subsubsection{Reagents and samples}

$\beta$-Mercaptoethanol was purchased from AMRESCO, The Netherlands. Taq DNA polymerase, dNTP and $100 \mathrm{bp}$ DNA Ladder were purchased from Shanghai Sangon Biological Engineering \& Technology services Co. Ltd. (SSBE), China. The streptavidin MicroBeads were purchased from MACS, Germany. TPA was purchased from Aldrich Chemical Company. GM soybeans (Brazil soybean No. 1, Brazil soybean No. 2 and Argentina soybean No. 1) were from Brazil and Argentina. Non-GM soybeans (yu soybean No. 1) were from China. GM papayas (Huanong-1) and non-GM papayas (Suizhonghong) were gifts from South China Agricultural University.

\subsubsection{Primers and probes}

PCR primers (Lipp et al., 1999) and probes were all synthesized by SSBE (Table 1). The probes were labeled with biotin by SSBE or ruthenium by our lab, respectively.

\subsection{Methods}

\subsubsection{Principle}

The basic principle of the assay was outlined in Fig. 3. PCR amplifications for soybeans and papayas were performed according to the IUPAC method that has been used for GMOs detection (Lipp et al., 1999). Almost all GM soybeans and papayas contain the Cauliflower Mosaic Virus promoter (P-CaMV35S) (Ahmed, 2002; Gachet et al., 1999). We designed a pair of primers to amplify a 195bp fragment in the P-CaMV35S. So, the fragment would be amplified from GMOs instead of non-GMOs through PCR (sometimes, nonspecific amplification would occur). After PCR, 
Table 1

Primers and probes used in the study

\begin{tabular}{llr}
\hline Name & Sequence $\left(5^{\prime}-3^{\prime}\right)$ & Product size (bp) \\
\hline $35 \mathrm{~S}$ sense primer & getcctacaatgccatca & $195($ sense primer + antisense primer $)$ \\
$35 \mathrm{~S}$ antisense primer & gatagtgggattgtgcgtca & \\
$35 \mathrm{~S}$ probe 1 & cggcagaggcatcttcaacgatggcc-biotin & \\
$35 \mathrm{~S}$ probe 2 & Ru-tttcacgatgctctcgtgggtggg & \\
\hline
\end{tabular}

the products would hybridize with a pair of oligonucleotide probes. They are designed to hybridize with the $195 \mathrm{bp}$ fragment. Nonspecific amplified products could not hybridize with the probes. One of the probes was labeled by biotin, but another was labeled by ruthenium. The biotin labeled DNA was bound to the surface of streptavidin-coupled beads through the highly selective biotin-streptavidin linkage. The unlinked DNA fragments were washed away. The TBR labeled with the probe would emit light on the anode surface. The light would be recorded as an ECL signal, which reflects the quantity of the hybridized PCR products. Finally, we could confirm whether GM components existed.

\subsubsection{DNA extraction}

The cetyltrimethyl ammonium bromide (CTAB) method for sample extraction and purification reported by Lipp et al. (1999) was used in this study. The samples with or without GM components were minced with sterile surgical blades and dry samples as flour are moistened with the three-fold amount of water. Then they were extracted with CTAB, precipitated, treated with chloroform, and precipitated with isopropanol to obtain a purified DNA matrix.

\subsubsection{PCR amplification}

DNA from GM soybeans and papayas and non-GM soybeans and papayas were amplified following the procedure reported by Pietsch et al. (1997). The thermocycler (PTC-100 MJ Research Inc., USA) was programmed with an initial step of denaturation at $94^{\circ} \mathrm{C}$ for $3 \mathrm{~min}$. Cycling conditions were: denaturation at $94{ }^{\circ} \mathrm{C}$ for $20 \mathrm{~s}$, anneal at

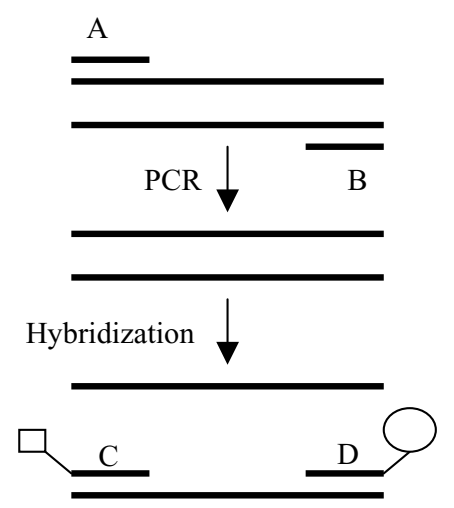

Fig. 3. Basic principle of GMO detection. (A and B) primer; (C) biotin-labeled probe; (D) ruthenium-labeled probe; $(\square)$ biotin; $(\bigcirc)$ ruthenium.
$54{ }^{\circ} \mathrm{C}$ for $40 \mathrm{~s}$ and elongation at $72{ }^{\circ} \mathrm{C}$ for $1 \mathrm{~min}$. In total, 40 cycles of above program were performed. The last round of elongation was for $3 \mathrm{~min}$. From the amplification of the DNA regions, fragment of 195 base pairs (bp) was obtained. The control solution (blank) contained all the PCR regents except the DNA template.

\subsubsection{Hybridization with a pair of oligonucleotide probes}

Hybridizations with biotin labeled probe and TBR labeled probe were performed by adding $20 \mu \mathrm{l}$ of each to $20 \mu \mathrm{l}$ of PCR products. The mixture was incubated for $5 \mathrm{~min}$ at $95^{\circ} \mathrm{C}$ and $10 \mathrm{~min}$ at $65^{\circ} \mathrm{C}$ in the PCR system (PTC-100 MJ, USA) (Jong et al., 2000).

\subsubsection{ECL detection}

Twenty $\mu \mathrm{l}$ of hybridization products was added to $20 \mu \mathrm{l}$ of binding buffer. The solution was incubated at room temperature for $10 \mathrm{~min}$. Then, $10 \mu \mathrm{l}$ of streptavidin-coated magnetic beads was added. The mixture was then shaken at room temperature for $20 \mathrm{~min}$. After washing and removing the supernatant, the sample was added to the flow ECL detection cell. Then, TPA was added to the reaction cell. A voltage of $1.25 \mathrm{~V}$ was applied across the electrodes and the signals of ECL were measured by PMT. At last, computer read the ECL signals by labview software. Each sample was detected 10 times and analyzed with statistical method.

\section{Results}

\subsection{Electrophoresis analysis for PCR products}

To verify the feasibility of the method, $2 \%$ agarose gel electrophoresis analysis for PCR products was performed in the experiment. As shows in Fig. 4, three bands of $195 \mathrm{bp}$ appear in the lanes of three kinds of GM soybeans PCR products, while no PCR amplification detected in negative control and non-GM soybeans. The results of gel electrophoresis are consistent with the results of ECL detection.

\subsection{Capability of ECL detection system}

The calibration curve (Fig. 5) was obtained by measuring different quantity of labeled DNA. The minimum detectable quantity was $100 \mathrm{fmol}$. The curve shows a profile with a linear region from 0.1 to $250 \mathrm{pmol}\left(R^{2}=0.997\right)$. This wide dynamic range is useful in developing quantification assay. 


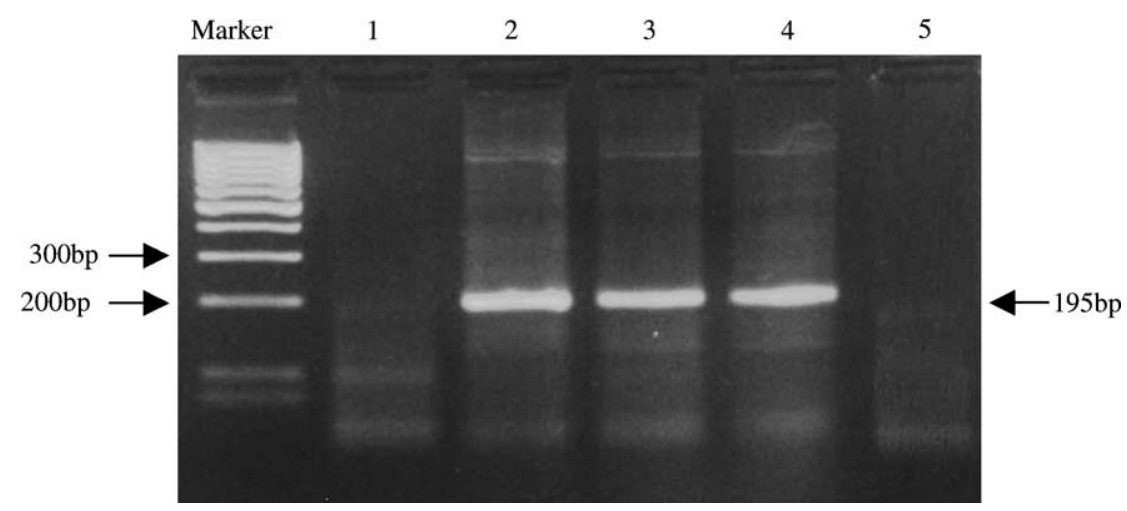

Fig. 4. PCR amplification of genetically modified soybeans DNA. Marker: 100 bp ladder; 1: non-GM soybeans (yu soybean No. 1); 2: GM soybeans (Brazil soybean No. 1); 3: GM soybeans (Brazil soybean No. 2); 4: GM soybeans (Argentina soybean No. 1); control: all the PCR regents except the DNA template.

In order to avoid cumulated background signals, the assay started from low quantity to high quantity. The ECL detection cell was cleaned by distilled water after detection.

\subsection{ECL detection results}

Fig. 6 shows the results of ECL detection for GM soybeans. The signals of blank control are $5.81 \pm 0.3 \mathrm{cps}$ (mean \pm standard deviation). And the signals of non-GM soybeans (yu soybean No. 1) are $12.8 \pm 1.9$. According to the data, we set the threshold as $18.5 \mathrm{cps}$ (mean of non-GMOs plus three times SD) to judge the negative. However, the signals of Brazil soybean No. 1, Brazil soybean No. 2 and Argentina soybean No. 1 are $207 \pm 10.4 \mathrm{cps}, 112 \pm 5.6 \mathrm{cps}$ and $83 \pm 4.2 \mathrm{cps}$, respectively. The signal-to-noise ratio of ECL detection was so great (signal-to-noise ratio: $\geq 6.5$ ) that we could confirm whether the samples have GM components by ECL intensity or nor.

Fig. 7 is the results of ECL detection for GM papayas (Huanong-1). The signals from control and non-GM papayas (Suizhonghong) are $5.3 \pm 0.3 \mathrm{cps}$ and $5.8 \pm 0.3 \mathrm{cps}$,

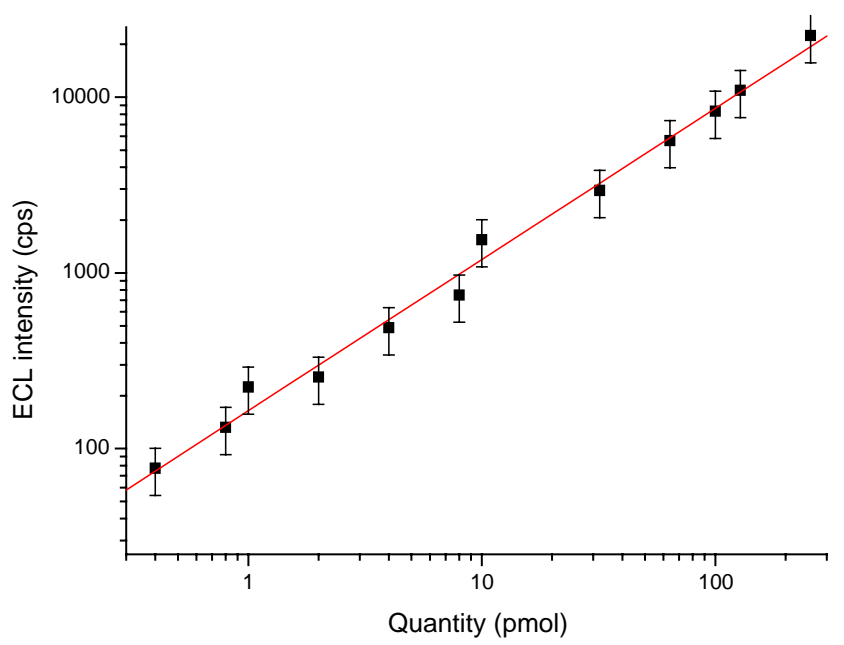

Fig. 5. Calibration curve for the ECL system.

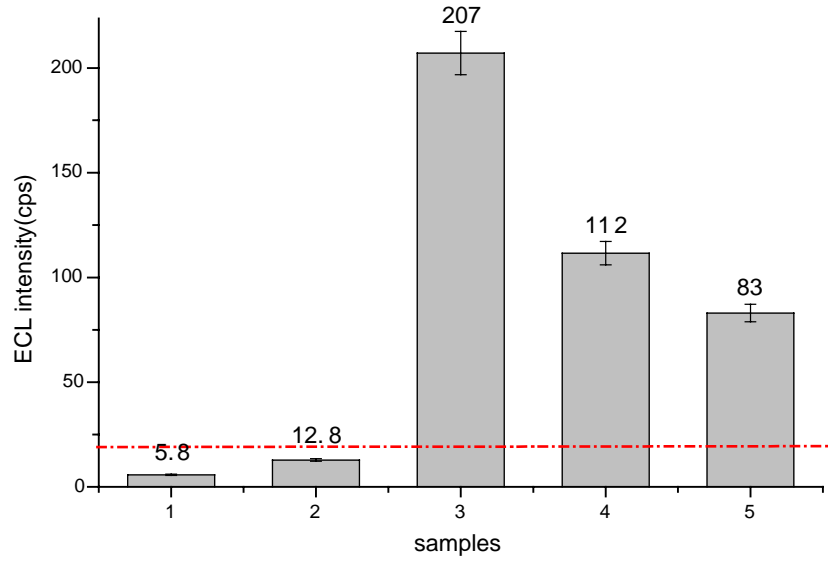

Fig. 6. Detection of genetically modified soybeans. 1: control (TE+TPA); 2: non-GM soybeans (yu soybean No. 1); 3: GM soybeans (Brazil soybean No. 1); 4: GM soybeans (Brazil soybean No. 2); 5: GM soybeans (Argentina soybean No. 1).

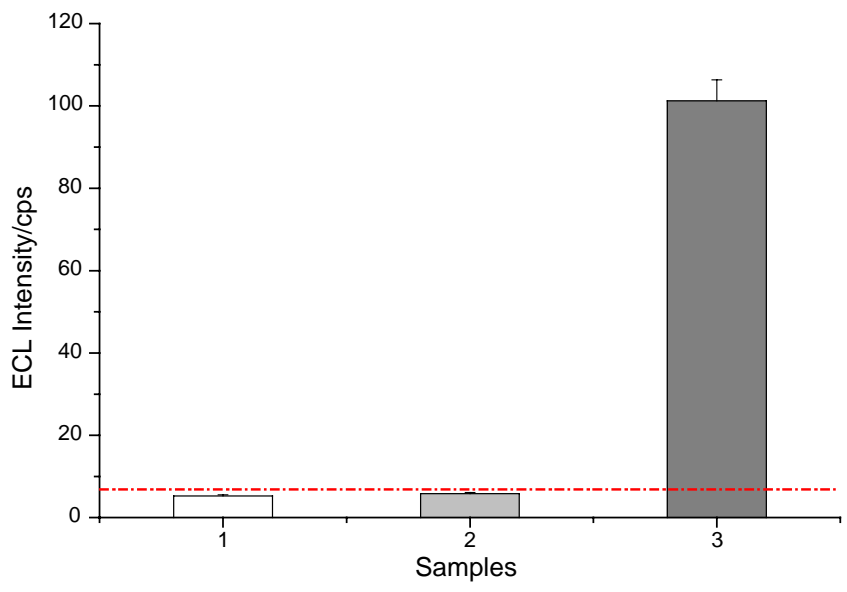

Fig. 7. The detection of genetically modified papayas. 1: control (TE + TPA); 2: non-GM papayas (Suizhonghong); 3: GM papayas (Huanong-1) 
respectively. The threshold was set as $6.7 \mathrm{cps}$. We consider the non-GM papayas were not detectable. The signal of GM papayas is $101.2 \pm 5.1 \mathrm{cps}$. The results strengthen the feasibility of the ECL-PCR detection for GMOs.

Based on the calibration curve, the detection products' quantity of Brazil soybean No. 1, Brazil soybean No. 2, Argentina soybean No. 1 and GM papayas (Huanong-1) are $1.27 \pm 0.07 \mathrm{pmol}, 0.60 \pm 0.03 \mathrm{pmol}, 0.42 \pm 0.02 \mathrm{pmol}$ and, $0.53 \pm 0.02$ pmol, respectively.

\section{Discussion}

The amplification products have a double helices structure. The double strands will be separated by thermally denaturing and hybridize with the pair of probes, which was designed for specific selection for CaMV35S promoter in GM soybeans and papayas. Streptavidin-coated magnetic beads could catch the specific PCR products, which has hybridized with the biotin labeled probe, through the biotin-streptavidin conjunction. TBR label will react with TPA at working voltage to emit light for detection. Thus, only the PCR products hybridized with both biotin-labeled and TBR-labeled probes could be detected by ECL assay. The false positive result caused by nonspecific amplification could be avoided, for the probes will not hybridize with the nonspecific amplified products.

In the early 1990s, Kenten and co-workers established the ECL method for nucleic acid analysis. With the rapid development of biotechnology, ECL method was widely used in gene analysis. But, up to now, ECL has not been used to detect GMOs. For the first time, ECL is combined with PCR and hybridization for GMOs detection. The high specificity was realized in our experiment result (Figs. 6 and 7). The results show that the ECL signals of non-GM soybeans and papayas are very low. So we consider they are undetectable. We set the threshold according to the data of known non-GMOs. However, the signals of four kinds of GM samples are far higher than the threshold value. The system has an excellent signal-to-noise ratio. Thus, the ECL-PCR method is feasible for detection for GMOs.

The method does not use any poisonous materials, such as ethidium bromide or isotopes. It provides an extremely sensitive detection at subpicomolar concentration, as well as a very wide dynamic range. Compared with gel electrophoresis analysis, it is no poisonous and easier to operate. In conclusion, the ECL-PCR could be a newly quantitative analysis method for GMOs detection.

\section{Conclusion}

In this paper, ECL-PCR has been applied to GMOs detection for the first time. The high specificity was realized by hybridization with a pair of probes labeled with biotin and TBR. The method can detect GMOs with high sensitiv- ity, wide dynamic range and rapidness. It could potentially become a rapid and convenient method for daily GMOs detection.

\section{Acknowledgements}

This research is supported by the National Natural Science Foundation of China (60378043), the Research Team Project of the Natural Science Foundation of Guangdong Province (015012), and the Project of Science and Technology of Guangdong Province (2002C20607) (principal).

\section{References}

Ahmed, F.E., 2002. Detection of genetically modified organisms in foods Trends Biotechnol. 20 (5), 215-223.

Blackburn, G.F., Shah, H.P., Kenten, J.H., Leland, J., Kamin, R.A., Link, J., Peterman, J., Powell, M.J., Shah, A., Talley, D.B., Tyagi, S.K., Wilkins, E., Wu, T.G., Massey, R.J., 1991. Electrochemiluminescence detection for development of immunoassays and DNA probe assays for clinical diagnostics. Clin. Chem. 37 (9), 1534-1539.

Council Regulation (EC) No. 49/2000 of the European Parliament and of the Council of 10 January 2000. See Official Journal L006, 11/01/2000, p. 0015.

Deaver, D.R., 1995. A new non-isotopic detection system for immunoassays. Nature 377 (6551), 758-776.

Droge, M., Puhler, A., Seilbitschka, W., 1998. Horizontal gene transfer as a biosafety issue: a natural phenomenon of public concern. J. Biotechnol. 64 (1), 75-90.

Gachet, E., Martin, G.G., Vigneau, F., Meyer, G., 1999. Detection of genetically modified organisms (GMOs) by PCR: a brief review of methodologies available. Trends Food Sci. Technol. 9, 380-388.

Hails, R.S., 2000. Reviews-genetically modified plants: the debate continues. Tree 15 (1), 14-18.

Hsueh, T.Y., Smith, R.L., Northrup, M.A., 1996. A microfabricated, electrochemiluminescence cell for the detection of amplified DNA. Sens. Actuators B 33, 110-114.

Jong, M.D., Weel, J.F.L., Schuurman, T., Dillen, P.M.E.W., Boom, R., 2000. Quantitation of varicella-zoster virus DNA in whole blood, plasma, and serum by PCR and electrochemiluminescence. J. Clin. Microbiol. 38 (7), 2568-2573.

Leland, J.K., Powell, M.J., 1990. Electrogenerated chemiluminescence: an oxidative-reduction type ECL reaction sequence using tripropylamine. J. Electrochem. Soc. 137, 3127-3131.

Lin, H.Y., Chang, J.W., Shih, D.Y.C., 2001. Detection of genetically modified soybeans by PCR method and immunoassay kits. J. Food Drug Anal. 9 (3), 160-166.

Lipp, M., Brodman, P., Pietsch, K., Pauwels, J., Anklam, E., 1999. IUPAC collaborative trial study of a method to detect genetically modified soybeans and maize in dried power. J. AOAC Int. 82, 923-928.

Mannelli, I., Minunni, M., Tombelli, S., Mascini, M., 2003. Quartz crystal microbalance (QCM) affinity biosensor for genetically modified organisms (GMOs) detection. Biosens. Bioelectron. 18, 129-140.

Mariotti, E., Minunni, M., Mascini, M., 2002. Surface plasmon resonance for genetically modified organism detection. Anal. Chim. Acta 453, $165-172$.

Minunni, M., Tombelli, S., Pratesi, S., Mascini, M., Piatti, P., Bogani, P., Buiatti, M., Mascini, M., 2001. A piezoelectric affinity biosensor for genetically modified organisms (GMOs) detection. Anal. Lett. 34, 6.

Niederhauser, C., Gilgen, M., Meyer, E., 1996. Gentechnologisch veranderte pflanzliche Lebensmittel: stand der anwendungsientiten Forschung und potentielle Nachweismoglichkeiten mit molekularbiologischen. Methoden. Mitt. Gebeite Lebensm. Hyg. 87, 307. 
Pietsch, K., Waiblinger, U., Brodman, P., Wurzl, A., 1997. Screeningverfahren zur identifizierung gentechnisch veranderter pflanzlicher lebensmittel. Deutsche Lebensmittel RundschauHelf 2, 35-38.

Vollenhofer, S., Burg, K., Schmidt, J., Kroath, H., 1999. Genetically modified organisms in food-screening and specific detection by polymerase chain reaction. J. Agric. Food Chem. 47 (12), 50385043.

Zhu, D.B., Xing, D., Shen, X.Y., Yan, G.H., 2003. High sensitive detection of presenilin-1 point mutation based on electrochemiluminescence. Chin. Sci. Bull. 48 (16), 1741-1744. 